\title{
INEXPENSIVE RF MODELING AND ANALYSIS TECHNIQUES AS APPLIED TO CYCLOTRONS
}

\author{
John Vincent \\ NSCL, East Lansing, MI 48824, USA
}

\begin{abstract}
A review and expansion of the circuit analogy method of modeling and analysing multiconductor TEM mode $\mathrm{rf}$ resonators is described. This method was used to predict the performance of the NSCL K500 and K1200 cyclotron resonators and the results compared well to the measured performance. The method is currently being applied as the initial stage of the design process to optimise the performance of the $\mathrm{rf}$ resonators for a proposed K250 cyclotron for medical applications. Although this technique requires an experienced $\mathrm{rf}$ modeller, the input files tend to be simple and small, the software is very inexpensive or free, and the computer runtimes are nearly instantaneous.
\end{abstract}

\section{THE MODELING TECHNIQUE}

The modeling technique applied is well known and fundamentally consists of slicing the TEM-mode resonator up into pieces that can be accurately described by equivalent linear circuit elements. The response of the resulting circuit is then equivalent to the response of the actual resonator within some predictable margin of error. To be successful, the modeler must meet two fundamental requirements; 1) the modeler must be able to qualitatively understand the standing-wave pattern of the structure and describe it terms of an equivalent circuit, and 2) the modeler must be able to determine accurate circuit models for the resonator elements.

The circuit elements used to model the resonator are linear and include resistors, capacitors, inductors, and transmission lines. The lumped elements are normally used to describe discontinuities and coupling elements while the transmission lines are used to describe distributed effects. In many cases, the transmission line segments of the circuit are both non-uniform (the transverse cross-section varies along the direction of wave propagation) and non-standard (the transverse crosssection does not have a handbook solution). Since the transmission line is normally the dominant element used in the model, the central issue to this method involves efficiently determining accurate representations of nonuniform and non-standard transmission line segments. This paper shall therefore describe in detail an accurate model for non-uniform, non-standard transmission lines.

\section{THE TRANSMISSION LINE MODEL}

The detailed derivations from first principles for the transmission line model described here as well as formulas for many other components of the equivalent circuit such as bends, discontinuities, couplers, etc. may be found in reference[1]. Formulas and techniques for standard components and systems can be found elsewhere[2]. The technique requires that numerical analysis be performed on the cross-section at each end of the transmission line segment to determine the transmission line parameters. Normally this analysis is performed with electrostatic software since the fundamental TEM mode supports this field solution, however, with the proper software this technique is extensible to higher order transmission line modes. The parameters thus obtained are fed to formula to parameterise a standard uniform transmission line of the same length with characteristic impedance and filler/conduction loss parameters in a manner that attempts to accurately represent the electric stored energy, magnetic stored energy and losses. The technique is extended in this paper to better maintain the stored energy through the addition of lumped elements expressly added for this purpose. The final circuit describing the segment thus accurately models the structure for stored energy (both electric and magnetic), time delay, and losses

Figure 1 illustrates the process of converting a mechanical segment of transmission line to its equivalent circuit illustration. It is assumed that the segment has been taken short enough such that the characteristic impedance can be assumed to vary linearly from "Face A" to "Face B". The conduction losses are maintained by using a formulation that maps the losses to an "Equivalent Width" of conductor (wi, wo) with constant current density. The constant characteristic impedance (Zo) of each line in the model is calculated based on a formulation that attempts to best account for the magnetic and electric stored energy. The lumped elements are used to account for the magnetic and electric energy left unaccounted for by the transmission line segments.

The first step in modeling a transmission line segment requires determining the parameters of each face. This is typically accomplished by invoking a numerical analysis program. A program that does a particularly 

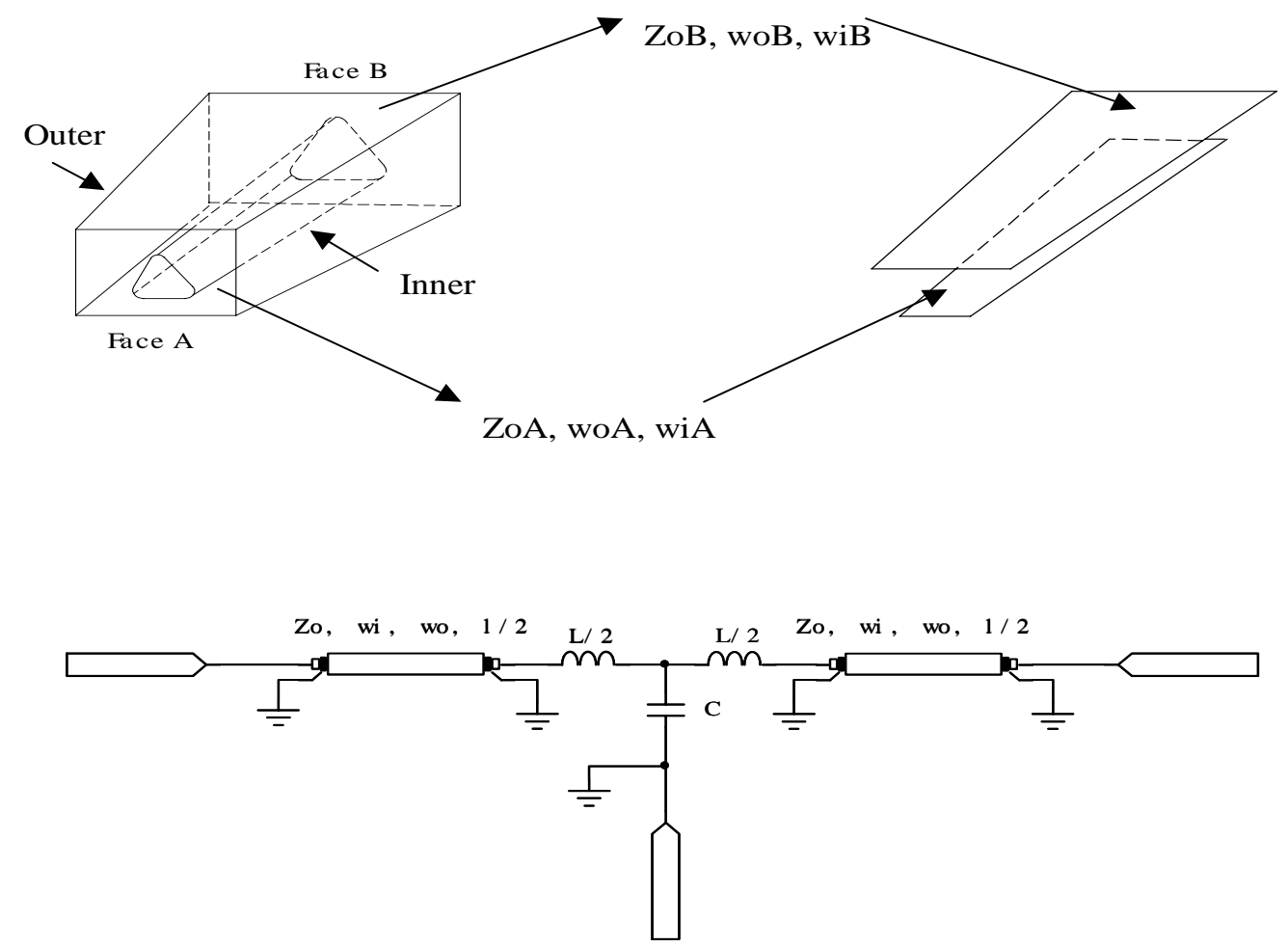

Figure 1: An illustration of the process of converting a mechanical segment of transmission line to its equivalent circuit representation.

good job for low cost is available from "PDE Solutions"[5]. An example of output from this software is shown in figure 2 . The example is an equipotential plot for a representative K250 upper dee cross-section.

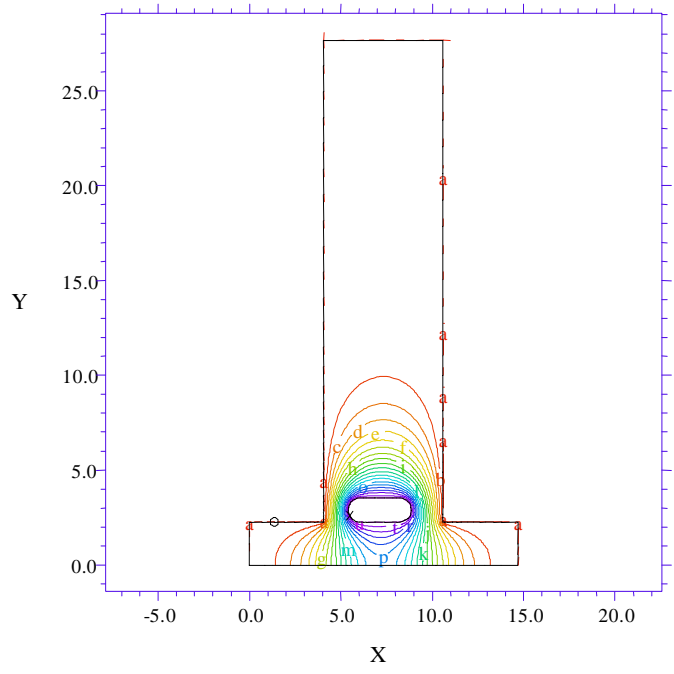

Figure 2: An example numerical analysis performed on a typical K250 cyclotron cross-section.

For the example shown in figure 2, the parameters describing the transmission line at this face are calculated using formula entered into this software to be: $\mathrm{Zo}=78.4$ Ohms, wi $=6.61 \mathrm{~cm}$, wo $=11.7 \mathrm{~cm}$. For reference, the actual boundary lengths are $8.36 \mathrm{~cm}$, and $84.8 \mathrm{~cm}$ for the inner and outer boundaries respectively. The i,o subscripts denote the inner, outer conductors.

\subsection{The Transmission Line Transformation}

To transform a non-uniform, non-standard transmission line to the equivalent circuit described, the following formulas are used. The multiple subscripts denote an analysis is performed independently for both the inner " $\mathrm{i}$ " and outer "o" conductors. An error free analysis would yield $\mathrm{Ci}=\mathrm{Co}$ and $\mathrm{Zoi}=\mathrm{Zoo}$. First each cross-section (A, B) is analysed to ascertain the following parameters. The subscript " $\mathrm{s}$ " denotes the field at the surface of the conductor. In the following formula, The "L" represents length and the "v" represents velocity. The capacitance (Farads/m) and charge (Colombs/m) are per unit length values. 


$$
\begin{gathered}
w_{i, o}=\frac{\left[\int_{0}^{l}\left|E_{s i, o}\right| d l\right]^{2}}{\int_{0}^{l}\left|E_{s i, o}\right|^{2} d l} \quad Q_{i, o}=\int_{0}^{l}\left|E_{s i, o}\right| d l \quad(\mathrm{C} / \mathrm{m}) \\
C_{i, o}=\frac{Q_{i, o}}{V_{i, o}}(\mathrm{~F} / \mathrm{m}) \quad Z_{o i o}=\frac{1}{v C_{Y, o}}(\mathrm{Ohms})
\end{gathered}
$$

The information obtained from the analysis of each crosssection is then used to determine the parameters of the equivalent circuit transmission lines with the following formula.

$$
\begin{gathered}
w_{e}=\frac{w_{A i, o}-w_{B i, o}}{\ln \left(\frac{w_{A i, o}}{w_{B i, o}}\right)}, Z_{o}=Z_{o A} \sqrt{\frac{\left(\tau^{2}-1\right)}{2 \ln (\tau)}}(\mathrm{Ohms}) \\
\tau=\frac{Z_{o B}}{Z_{o A}}
\end{gathered}
$$

The previous formula completely conserved the conduction losses, however, no choice of constant Zo can completely conserve the stored energy. The following formula for the lumped elements of the equivalent circuit regain any stored energy left unaccounted for.

$$
\begin{gathered}
C=C_{o A} l\left[\frac{\ln (\tau)}{\tau-1}-\sqrt{\frac{2 \ln (\tau)}{\tau^{2}-1}}\right] \\
L=L_{o A} l\left[\frac{\tau+1}{2}-\sqrt{\frac{1}{2} \frac{\left(\tau^{2}-1\right)}{\ln (\tau)}}\right]
\end{gathered}
$$

Using the above expressions, the original non-uniform and non-standard section of transmission line has been transformed to an equivalent circuit that conserves in the most balanced way the electrostatic and magnetostatic stored energy, all losses (conduction and material), and propagation delay of the original segment of line. The graphs in figure 3 may be used in lieu of the equations to determine the values of elements of the equivalent circuit.
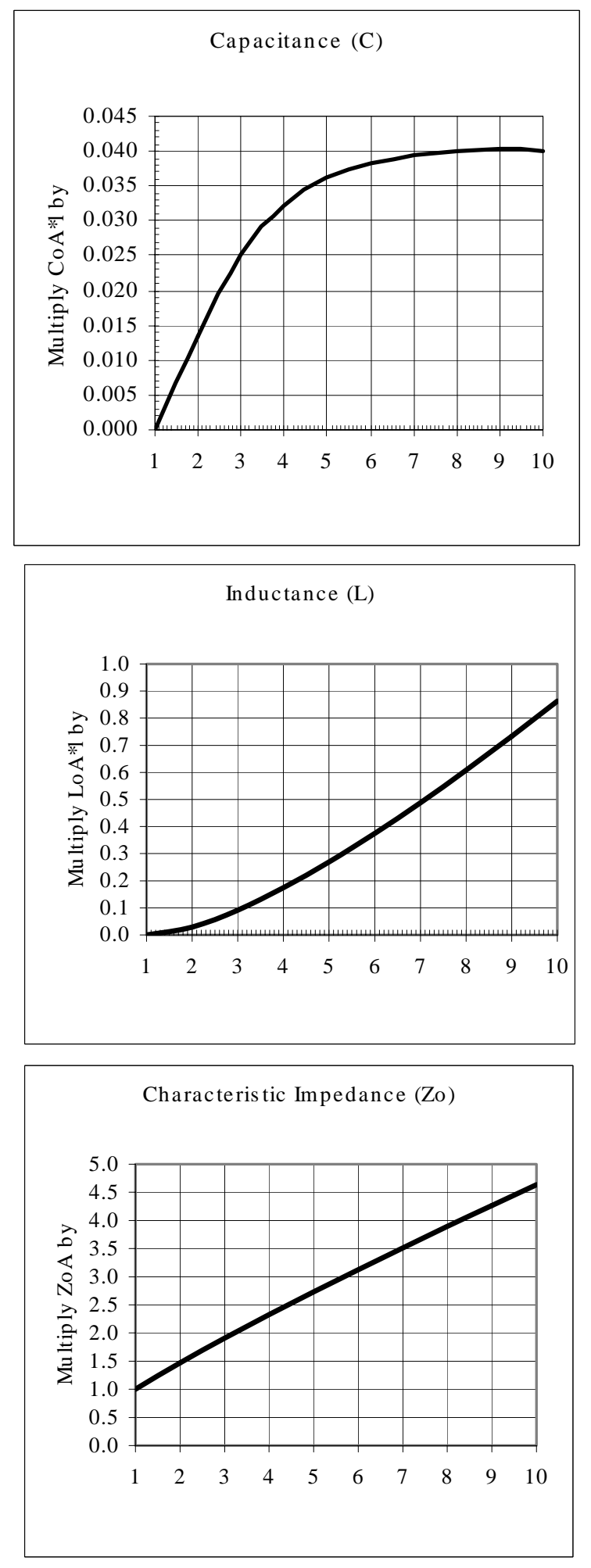

Figure 3: Graphs used to determine the values for the elements of the equivalent circuit in lieu of applying the formula. 


\section{APPLICATION}

This method was applied to the analysis of the K1200 cyclotron resonators[1], and the recent upgrade of the K500 resonators[3]. The results of these analysis compared excellently with the measured response. The technique and associated software and is now being used as the preliminary step to optimise and predict the performance of the resonators for a K250 cyclotron intended for proton therapy cyclotron[4].

For the K250, the software now being used to analyse the non-standard cross-sections is called "FlexPDE" and is available from PDE Solutions Inc.[5]. The software used to analyse the resultant circuits is called WAC and is available from the author's business Precision Power Products[6]. Both companies offer a free version available for download. Both packages come with online documentation and numerous examples. PDE Solutions also sells books that have applied the software to many areas of physics[7,8]. Copies of both programs have been included on the $\mathrm{CD}$ for this conference.

The accuracy of the model is certainly dependent on the modeller's understanding, however, assuming a competent modeller, the accuracy of the results is reasonably predictable. It is normally obvious what elements of the circuit have accurate models and what elements have debatable models. The magnitude of the response of the circuit to an error in some particular circuit element value is a function of how large a fraction of the overall circuit energy or losses the element manages. Hence large uncertainty in elements that manage small amounts of the overall loss or energy will have small effects on the resonant frequency or overall losses. Conversely, large uncertainty in elements that manage large amounts of energy results in a large error. Using this knowledge and a qualitative estimate of the uncertainty for each circuit element, a quantitative estimate of the expected circuit accuracy can be calculated[1].

\section{CONCLUSION}

The method of representing complex multiconductor resonators by an equivalent circuit is a powerful technique that should not be abandoned. Although 3-demensional partial differential equation solvers do now exist from multiple vendors, costs tend to be high, inputs tend to be complex, and runtimes tend to be long. Simple changes for optimisation runs can take large input efforts and long periods between trials. It is a good alternative to use the equivalent circuit technique for initial design, optimisation and understanding followed (when possible ) by a 3-D solver analyses to check the result. However, nothing can beat measurements on an actual physically built model as the definitive source of information whether it be sought or unexpectedly discovered!

\section{REFERENCES}

[1] J. Vincent, "Modeling and Analysis of RF Structures using an Equivalent Circuit Methodology with Application to Charged Particle Accelerator RF Resonators", UMI \# 9631355, UMI Company, Ann Arbor, MI, 48103, 1996, Internet. Available from http://www.3pco.com/content/Thesis/Thesis.pdf; accessed April 16, 2001

[2] V. Valkenburg, "Reference Data for Engineers: Radio, Electronics, Computer \& Communications", Eight Edition, Sams Publishing, June 1993.

[3] T. Berenc, J. Vincent, Rfnote \#116, "CCP K500 Tuning Stem Design”, 1996, Internet. Available from http://www.nscl.msu.edu/departments/electronics/RF Notes ; accessed 16 April, 2001

[4] J. Vincent, Rfnote \#122, "K250 RF Analysis", 2001, Internet. Available from http://www.nscl.msu.edu/departments/electronics/RF Notes ; accessed 16 April, 2001

[5] FlexPDE software, PDE Solutions inc, Internet. Available from http://www.flexpde.com/ ; accessed 16 April, 2001.

[6] WAC software, Precision Power Products, Internet. Available from http://www.3pco.com/ ; accessed 16 April, 2001.

[7] G Backstrom, "Fields of Physics by Finite Element Analysis An Introduction”, ISBN 91-44-00655-1, 1998, Internet. Available from http://www.flexpde.com/; accessed 16 April, 2001.

[8] G Backstrom, "Waves by Finite Element Analysis", ISBN 91-44-01007-9, 1999, Internet. Available from http://www.flexpde.com/; accessed 16 April, 2001. 